\title{
Phylogeny of Rickettsia spp. inferred by comparing sequences of 'gene $D$ ', which encodes an intracytoplasmic protein
}

Unité des Rickettsies, Faculté de Médecine, CNRS UPRES-A 6020, Université de la Méditerranée, 27 bd J. Moulin, 13385 Marseille Cedex 05, France

\author{
Zuzana Sekeyova, † Véronique Roux and Didier Raoult
}

Author for correspondence: Didier Raoult. Tel: +334913243 75. Fax: +33491830390. e-mail: Didier.Raoult@medecine.univ-mrs.fr

\begin{abstract}
'Gene D' is the PS120-protein-encoding gene, first described in Rickettsia conorii and Rickettsia japonica. Sequence analysis of a 3030 bp fragment of ' gene D' in 24 representatives of the genus Rickettsia was carried out to complete phylogenetic analyses previously inferred by comparison of gene sequences encoding citrate synthase, $17 \mathrm{kDa}$ antigen and rOmpA and rOmpB. The phylogenetic relationships between rickettsiae were inferred from the comparison of both the gene and the derived protein sequences, using the parsimony, neighbour-joining and maximum-likelihood methods. Five distinct groups of rickettsiae were identified. These were: the Rickettsia massiliae group, including R. massiliae, Bar 29, Rickettsia rhipicephali and Rickettsia aeschlimannii; the Rickettsia rickettsii group containing Rickettsia sibirica, 'Rickettsia mongolotimonae', Rickettsia parkeri, strain S, Rickettsia africae, the R. conorii complex, Rickettsia slovaca, Rickettsia honei, $R$. rickettsii, $R$. japonica and Rickettsia montanensis; the group currently containing only Rickettsia helvetica; the Rickettsia akari group including Rickettsia australis, $R$. akari and the ELB agent; Rickettsia prowazekii and Rickettsia typhi clustered in the typhus group. As significant bootstrap values were obtained for most of the nodes, sequence comparison of ' gene D' should be considered as a complementary approach in phylogenetic studies of rickettsiae.
\end{abstract}

Keywords: gene D, Rickettsia, phylogenetic analysis

\section{INTRODUCTION}

Rickettsia are obligate intracellular Gram-negative bacteria and although arthropods are more often their main reservoir hosts, people and other vertebrates may also be infected. Comparative microimmunofluorescence (MIF) testing with polyclonal mouse sera (Philip et al., 1978; Beati et al., 1994; Eremeeva et al., 1995) has long been the reference method for the identification of rickettsiae but genotypic identification is now used more frequently. Early reports showed interspecies differences using RFLP analysis of PCRamplified DNA (Regnery et al., 1991; Eremeeva et al., 1994). Later, significant differences in the $16 \mathrm{~S}$ rRNA

\footnotetext{
† Present address: Institute of Virology, Slovak Academy of Sciences, Dubravska cesta, 9, 84246 Bratislava, Slovak Republic.

Abbreviations: SFG, spotted fever group; TG, typhus group.

The GenBank accession numbers for the nucleotide sequences reported in this paper are given in Methods.
}

gene sequences of members of the genus Rickettsia resulted in its division into two genera. The first one was the newly created genus Orientia (Tamura et al., 1995) and the other was the genus Rickettsia, which was itself subdivided in two groups. The typhus group (TG) included Rickettsia prowazekii, Rickettsia typhi and Rickettsia canadensis while the spotted fever group (SFG) included all other rickettsiae. Recent phylogenetic studies of rickettsiae using genes encoding 16S rRNA (Stothard \& Fuerst, 1995; Roux \& Raoult, 1995), citrate synthase (gltA) (Roux et al., 1997), $17 \mathrm{kDa}$ antigen (Stenos et al., 1998; Anderson et al., 1988), rOmpA (Fournier et al., 1998) and rOmpB (Roux \& Raoult, 2000) have suggested a different organization for the genus. Several distinct clusters of organisms were shown to occur in the SFG, indicating the heterogeneity of the genus. Moreover, $R$. canadensis and Rickettsia bellii were shown to be sufficiently distinct to warrant their removal from the TG and SFG, respectively. As new rickettsiae continue to be identified, it is important to select informative 
Table 1. List of the strains included in the study

\begin{tabular}{|c|c|c|}
\hline Rickettsia & Strain & $\begin{array}{l}\text { Size of the sequenced } \\
\text { gene fragment (bp) }\end{array}$ \\
\hline R. aeschlimannii & $\mathrm{MC} 16^{\mathrm{T}}$ & 3010 \\
\hline R. africae & EFS-5 & 3034 \\
\hline Astrakhan fever rickettsia & A-167 & 3028 \\
\hline R. australis & Phillips & 2999 \\
\hline R. akari & MK, ATCC VR-148 & 2995 \\
\hline Bar 29 & Bar 29 & 3034 \\
\hline R. conorii & Moroccan, ATCC VR-141 & 3028 \\
\hline R. conorii & Indian tick typhus rickettsia, ATCC VR-597 & 3028 \\
\hline ELB bacterium & $\mathrm{URRWX} \mathrm{Cal}_{2}$ & 2991 \\
\hline R. helvetica & C9P9 & 3028 \\
\hline R. honei & Thai tick typhus rickettsia, ATCC VR-599 & 3034 \\
\hline Israeli tick typhus rickettsia & ISTT CDC1 & 3028 \\
\hline R. japonica & YM & 3016 \\
\hline R. massiliae & Mtu1 $^{\mathrm{T}}$ & 3031 \\
\hline ' $R$. mongolotimonae' & HA-91 & 3034 \\
\hline R. montanensis & $\mathrm{M} / 5-6$ & 3028 \\
\hline R. parkeri & Maculatum 20 & 3031 \\
\hline R. prowazekii & Breinl, ATCC VR-142 & 3018 \\
\hline R. rhipicephali & $3-7-6$ & 3040 \\
\hline R. rickettsii & R (Bitter root), ATCC VR-891 & 3022 \\
\hline Strain S & Strain $\mathrm{S}$ & 3034 \\
\hline R. sibirica & 246, ATCC VR-151 & 2974 \\
\hline R. slovaca & $13-\mathrm{B}$ & 3037 \\
\hline R. typhi & Wilmington, ATCC VR-144 & 3022 \\
\hline
\end{tabular}

gene sequences to establish phylogenetic analysis and classification of these organisms.

'Gene D' is a recently described gene in rickettsiae and its potential for facilitating phylogenetic analysis of these bacteria has yet to be considered. It was first identified by Schuenke \& Walker (1994) from three overlapping constructs derived from genomic Rickettsia conorii DNA libraries. Sequencing revealed it to be a 3065 nucleotide open reading frame encoding a protein which was recognized in humoral and cellmediated immune responses and was thus suspected of playing a role in protective immunity (Schuenke \& Walker, 1994). The sequencing data also showed that 'gene D' had no significant homology with any previously sequenced genes. Later, a major part of the corresponding 'gene D' of Rickettsia japonica was cloned and recombinant protein was expressed in Escherichia coli (Uchiyama et al., 1996). Antisera against the recombinant protein were found to react with the heat-stable 'PS120' proteins of $R$. japonica (Uchiyama et al., 1996). These proteins are distinct from the rOmpB outer-membrane protein (Carl et al., 1990) and, by immunoelectron microscopy, are found outside the electron-lucent nucleoid-like region in the cytoplasm of $R$. japonica (Schuenke \& Walker, 1994; Uchiyama, 1997).

In this report, we describe the sequencing of 'gene D' in 24 representatives of the genus Rickettsia and the phylogenetic data obtained by comparison of these sequences.

\section{METHODS}

Rickettsial strains. The strains used are listed in Table 1.

Rickettsial cultivation. The SFG and TG rickettsiae were grown on Vero cell monolayers as previously described (Roux et al., 1997). When Gimenez staining showed the cells to be heavily infected, they were harvested and centrifuged at $12000 \mathrm{~g}$ for $10 \mathrm{~min}$, resuspended in medium and stored at $-70{ }^{\circ} \mathrm{C}$ until purification of nucleic acid was carried out.

Nucleic acid purification, PCR amplification and sequencing reactions. Genomic DNA was extracted using Qiagen columns (QIAamp tissue kit; Qiagen) according to the manufacturer's instructions. PCR amplification and the sequencing reactions were performed using the oligonucleotide primers listed in Table 2 . The first 10 primers in the table were identified from sequence data on $R$. conorii (GenBank accession no. U01133) and used to amplify 'gene D' in five fragments (from I to V), each of a size suitable for subsequent sequencing. These primers amplified the gene in all the rickettsiae studied apart from Rickettsia akari, Rickettsia australis, ELB agent, R. prowazekii and $R$. typhi (see later). Specific primers D3064RICr and D3073JAPr were selected to amplify the $3^{\prime}$ end of the gene in Rickettsia rickettsii and $R$. japonica. A mutation in the 19 th position of the primer D2338f explained the necessity to modify the sequence of this primer for Rickettsia massiliae, Bar 29 and Rickettsia rhipicephali. The specific primers D1246HELf 


\begin{tabular}{|c|c|c|c|c|}
\hline Primer & Nucleotide sequence $5^{\prime} \rightarrow 3^{\prime}$ & Position in the gene & Primer used for: & Fragment \\
\hline D1f & ATG AGT AAA GAC GGT AAC CT & $1-20$ & Most of the rickettsiae & I \\
\hline D928r & AAG CTA TTG CGT CAT CTC CG & $928-907$ & Most of the rickettsiae & I \\
\hline D767f & CGA TGG TAG CAT TAA AAG CT & $767-786$ & Most of the rickettsiae & I \\
\hline D1390r & CTT GCT TTT CAG CAA TAT CAC & $1390-1370$ & Most of the rickettsiae & II \\
\hline D1219f & CCA AAT CTT CTT AAT ACA GC & $1219-1237$ & Most of the rickettsiae & II \\
\hline D1876r & TAG TTT GTT CTG CCA TAA TC & $1876-1857$ & Most of the rickettsiae & III \\
\hline D1738f & GTA TCT GAA TTA AGC AAT GCG & $1738-1758$ & Most of the rickettsiae & III \\
\hline D2482r & CTA TAA CAG GAT TAA CAG CG & $2482-2463$ & Most of the rickettsiae & IV \\
\hline $\mathrm{D} 2338 \mathrm{f}$ & GAT GCA GCG AGT GAG GCA GC & $2338-2357$ & Most of the rickettsiae & IV \\
\hline D3069r & TCA GCG TTG TGG AGG GGAA G & 3069-3050 & Most of the rickettsiae & $\mathrm{V}$ \\
\hline D547r & TAA ATC CAC CAG GGT ATC CA & $547-528$ & Most of the rickettsiae & $\mathrm{V}$ \\
\hline D2762f & CTA AAG AGG TAC GTT TAC AAA C & $2762-2783$ & Most of the rickettsiae & $\mathrm{V}$ \\
\hline D3064RICr & GTT GTG GAG GGG AAG ACT CTC & $3064-3044$ & R. rickettsii & $\mathrm{V}$ \\
\hline D3073JAPr & TTG ATC AGC GTT GTG GAG GG & $3073-3054$ & R. japonica & $\mathrm{V}$ \\
\hline D2338MBRf & GAT GCA GCG AGT GAG GCA AC & $2357-2338$ & R. massiliae, Bar 29, R. rhipicephali & $\mathrm{V}$ \\
\hline D1246HELf & TTA TCA GGC AGC ATG CAA GA & $1246-1265$ & R. helvetica & III \\
\hline D1862HELr & GTA ATC TTA TCT AAA TGC TGC & $1862-1842$ & R. helvetica & III \\
\hline D1652SIBf & CAA TTG ACG GTA TTA AGG ATG & $1652-1672$ & R. sibirica & IV \\
\hline D727CONf & CCT ATA AAA CTT GAT AAA GCC & $727-747$ & R. akari group and TG & \\
\hline $\mathrm{D} 791 \mathrm{CONr}$ & CCA TCA GCT TTT AAT GCT AC & $791-772$ & R. akari group and $\mathrm{TG}$ & \\
\hline D1605CONf & AGT AGA TGT CGG TTT AAG T & $1605-1623$ & R. akari group and $\mathrm{TG}$ & \\
\hline $\mathrm{D} 1835 \mathrm{CONr}$ & AAT TGT TGT TGT KCC GGA CT & $1816-1835$ & R. akari group and TG & \\
\hline DGW238AUSr & GGT CTG ATG TCT GAC CGT CA & $238-219$ & R. australis & \\
\hline DM67AAf & TTA CTT ACA GTT TGT GCT AA & M67-M47 & R. australis, R. akari & \\
\hline D328AAEf & GAC CGT GAT TTA GCT GAA CA & $328-347$ & R. australis, R. akari, ELB bacterium & \\
\hline D347AAEr & TGT TCA GCT AAA TCA CGG TA & $347-328$ & R. australis, R. akari, ELB bacterium & \\
\hline D1204AAEf & TCG CAA CAA GTG AAT CCA AA & $1204-1223$ & R. australis, R. akari, ELB bacterium & \\
\hline D1357AAEr & TAA GAA TTG CNG TAG CTG CT & $1357-1338$ & $R$. australis, $R$. akari, ELB bacterium & \\
\hline DGW1967AUSf & CAA AGG TTT TAG ATR GTC CG & $1967-1986$ & R. australis & \\
\hline DGW2344AUSf & GCG AGT GAG GYA GCT TTA GAT & $2344-2364$ & R. australis & \\
\hline DGW2594AUSf & ATT TTC GTG CTA TGC TYT CT & $2594-2613$ & R. australis & \\
\hline D2996AKAr & CTT GCA TTC ATT AAT GCT CC & $2996-2977$ & R. akari & \\
\hline D2077AAEr & CAA TGT CTG CTT TAT CTT G & $2077-2058$ & R. australis, R. akari, ELB bacterium & \\
\hline D2406AAEr & ATT CTG TCC TTT TAA CCC TT & $2406-2387$ & R. australis, $R$. akari, ELB bacterium & \\
\hline D2684AAEr & TTT ACC AGC TCA ACT ACT TT & $2684-2665$ & R. australis, $R$. akari, ELB bacterium & \\
\hline D51ELBf & TAA GGA ATA TAC AGA AGA A & $51-69$ & ELB bacterium & \\
\hline $\mathrm{D} 3022 \mathrm{ELBr}$ & TTA AAT TTT CCG ACT GAA TG & $3022-3003$ & ELB bacterium & \\
\hline DGW321TPf & TAA TAC AAA TCC AGA ACT MGC TG & $321-343$ & R. prowazekii, R. typhi & \\
\hline DGW343TPr & CAG CKA GTT CTG GAT TTG TAT TA & $343-321$ & R. prowazekii, R. typhi & \\
\hline DGW1141TPf & CAA GTG CCA CCA ATC ACT ACG & $1141-1161$ & R. prowazekii, R. typhi & \\
\hline DGW1161TPr & CGT AGT GAT TGG TGG CAC TTG & $1161-1141$ & R. prowazekii, R. typhi & \\
\hline DGW2069TPf & CAG AGA TTG TTA AAG GTA TAG G & $2069-2090$ & R. prowazekii, R. typhi & \\
\hline DGW2090TPr & CCT ATA CCT TTA ACA ATC TCT G & $2090-2069$ & R. prowazekii, R. typhi & \\
\hline DGW2444TPf & AAA CGC AAG ATG TWG CTA ATG & $2444-2464$ & R. prowazekii, R. typhi & \\
\hline DGW2464TPr & CAT TAG CWA CAT CTT GCG TTT & $2464-2444$ & R. prowazekii, R. typhi & \\
\hline
\end{tabular}


and D1862HELr were used to amplify fragment III of the Rickettsia helvetica gene and primer D1652SIBf was used in place of D1738f for the amplification of Rickettsia sibirica.

Each PCR was carried out in a Peltier model PTC-200 thermal cycler (MJ Research). Five microlitres of the purified DNA was amplified in a $50 \mu$ reaction mixture containing 25 pmol of each primer, $200 \mathrm{mM}$ (each) dATP, dCTP, dGTP and dTTP (Gibco-BRL Life Technologies), $1 \mathrm{U}$ ELONGASE (Gibco), $2 \mu \mathrm{l}$ buffer A and $8 \mu \mathrm{l}$ buffer B. Amplification was carried out with an initial $3 \mathrm{~min}$ denaturation at $95^{\circ} \mathrm{C}$ followed by 40 cycles of denaturation at $95^{\circ} \mathrm{C}$ for $30 \mathrm{~s}$, annealing at $50{ }^{\circ} \mathrm{C}$ for $30 \mathrm{~s}$ and extension at $68^{\circ} \mathrm{C}$ for $1 \mathrm{~min} 30 \mathrm{~s}$. The amplification was completed by holding the reaction mixture for $7 \mathrm{~min}$ at $68^{\circ} \mathrm{C}$ to allow complete extension of the PCR products.

PCR products were purified using a QIAquick Spin PCR purification kit (Qiagen) as described by the manufacturer. Sequencing reactions were carried out using a DNA sequencing kit (dRhodamine Terminator cycle sequencing ready reaction with Amplitaq Polymerase FS) (PE Applied Biosystems) as described by the manufacturer. Sequence products were purified and resolved on $5 \%(\mathrm{w} / \mathrm{v})$ polyacrylamide gels (Long Ranger Singel packs, Type $377-36 \mathrm{~cm}$ WTR) (Tebu) and electrophoresis was performed with the ABI PRISM 377 DNA sequencer (Perkin Elmer). The same primers as those used for PCR amplification were used for the sequencing reactions. Primers D547r and D2762f were used to confirm the sequence of the $5^{\prime}$ and $3^{\prime}$ end of the gene, respectively.

No PCR amplification was obtained with the primer pairs described above for the other rickettsiae. The rickettsial sequences obtained were aligned and two consensus primers were selected, D727CONf and D1835CONr. PCR amplification was obtained for R. akari, R. australis and the ELB bacterium. An improved PCR method for walking in uncloned genomic DNA, using the Universal Genome Walker kit (CLONTECH Laboratories) (Siebert et al., 1995), had to be used for the determination of the sequence of $R$. australis. Primers D791CONr and DGW238AUSr were used to determine the $5^{\prime}$ end of the 'gene D' sequence, and the primers D1605CONf, DGW1967AUSf, DGW2344AUSf and DGW2594f to determine the $3^{\prime}$ end of the sequence. Primers DM67AAf and D791CONr and primers D1605CONf and D2996AKAr were used to amplify the $5^{\prime}$ and $3^{\prime}$ ends of the $R$. akari gene, respectively, while primers D51ELBf and D791CONr and primers D1605CONf and D3022ELBr were used to amplify the 5' and $3^{\prime}$ ends of the ELB bacterium gene, respectively. Primers D328AAEf, D347AAEr, D1204AAEf, D1357AAEr, D2077AAEr, D2406AAEr and D2684AAEr were determined to confirm the sequence of 'gene D' of $R$. akari, $R$. australis and the ELB bacterium.

Amplification of $R$. prowazekii and R. typhi was carried out with primers $\mathrm{D} 727 \mathrm{CONf}$ and $\mathrm{D} 1835 \mathrm{CONr}$ and primers D1141TPf and D1161TPr. The genome walker approach was then used. Primers D791CONr, DGW321TPf and DGW343TPr were incorporated in the PCR amplification and sequencing reactions to determine the $5^{\prime}$ end sequences while primers DGW2069TPf, DGW2090TPr, DGW2444TPf and DGW2464TPr were used to determine the $3^{\prime}$ end sequences.

Data analysis. The 'gene D' sequences were translated into protein sequences using PC/GENE software (IntelliGenetics). The nucleic and amino acid sequences were aligned using the multisequence alignment program CLUSTAL within the
BISANCE environment (Dessen et al., 1990). The percentages of similarity were determined using the PC/GENE software package. The phylogenetic relationships between the representatives of the genus Rickettsia studied were determined using the 3.4 version of the PHYLIP software package (Felsenstein, 1989). Distance matrices generated by DNADIST and PROTDIST were determined under the assumptions of Kimura (1980). These matrices were used to elaborate dendrograms using the neighbour-joining method (Saitou \& Nei, 1987). The data were also examined by using parsimony and maximum-likelihood methods (DNAPARS and PROTPARS, and DNAML in PHYLIP). A bootstrap analysis was performed to investigate the stability of the resulting trees. Bootstrap values were obtained for a consensus tree based on 100 randomly generated trees by using SEQBOOT and CONSENSE in the same package.

Nucleotide sequence accession number. The GenBank accession numbers for the nucleotide sequence data reported in this paper are as follows: Rickettsia aeschlimannii, AF163005; Rickettsia africae, AF151724; Astrakhan fever rickettsia, AF163007; R. australis, AF187982; R. akari, AF213016; Bar 29, AF155056; ELB bacterium, AF196973; $R$. helvetica, AF163009; Israeli tick typhus rickettsia, AF155058; R. japonica, AF155055; R. massiliae, AF163003; 'Rickettsia mongolotimonae', AF151725; Rickettsia montanensis, AF163002; Rickettsia parkeri, AF155059; R. prowazekii, AF200340; $R$. rhipicephali, AF155053; $R$. rickettsii, AF163000; strain S, AF163001; R. conorii strain Moroccan, AF163008; R. sibirica, AF155057; Rickettsia slovaca, AF155054; Rickettsia honei, AF163004; R. typhi, AF188482; $R$. conorii strain Indian tick typhus rickettsia, AF163005.

\section{RESULTS}

\section{PCR amplification and sequencing}

'Gene D' fragments were amplified from all the strains of Rickettsia studied except $R$. bellii (strain 369L42-1) and $R$. canadensis (strain 2678). For each species the sequence of both DNA strands was determined at least twice. The 'gene D' was sequenced between bases 21 and 3050 for most of the species with respect to the open reading frame of the published sequence of $R$. conorii. Identical sequences were obtained to those already published for $R$. conorii (GenBank accession no. RCU01133), $R$. japonica (AB003696) and $R$. prowazekii (AJ235272). Minor differences in the sequences of the Indian tick typhus and Moroccan strains of $R$. conorii were obtained. The highest percentage of similarity between different species was obtained for the Israeli tick typhus rickettsia and the Astrakhan fever rickettsia (99.7\% for DNA sequences and $99.5 \%$ for protein sequences) and the lowest similarity was between $R$. prowazekii and $R$. akari (76.3\% for DNA sequences and $59.7 \%$ for protein sequences).

\section{Phylogeny inference}

Nucleotidic 'gene D' sequences were considered from base 53 to base 3006 to infer phylogenetic analysis. The dendrograms obtained with the three different treebuilding analysis methods used showed similar 


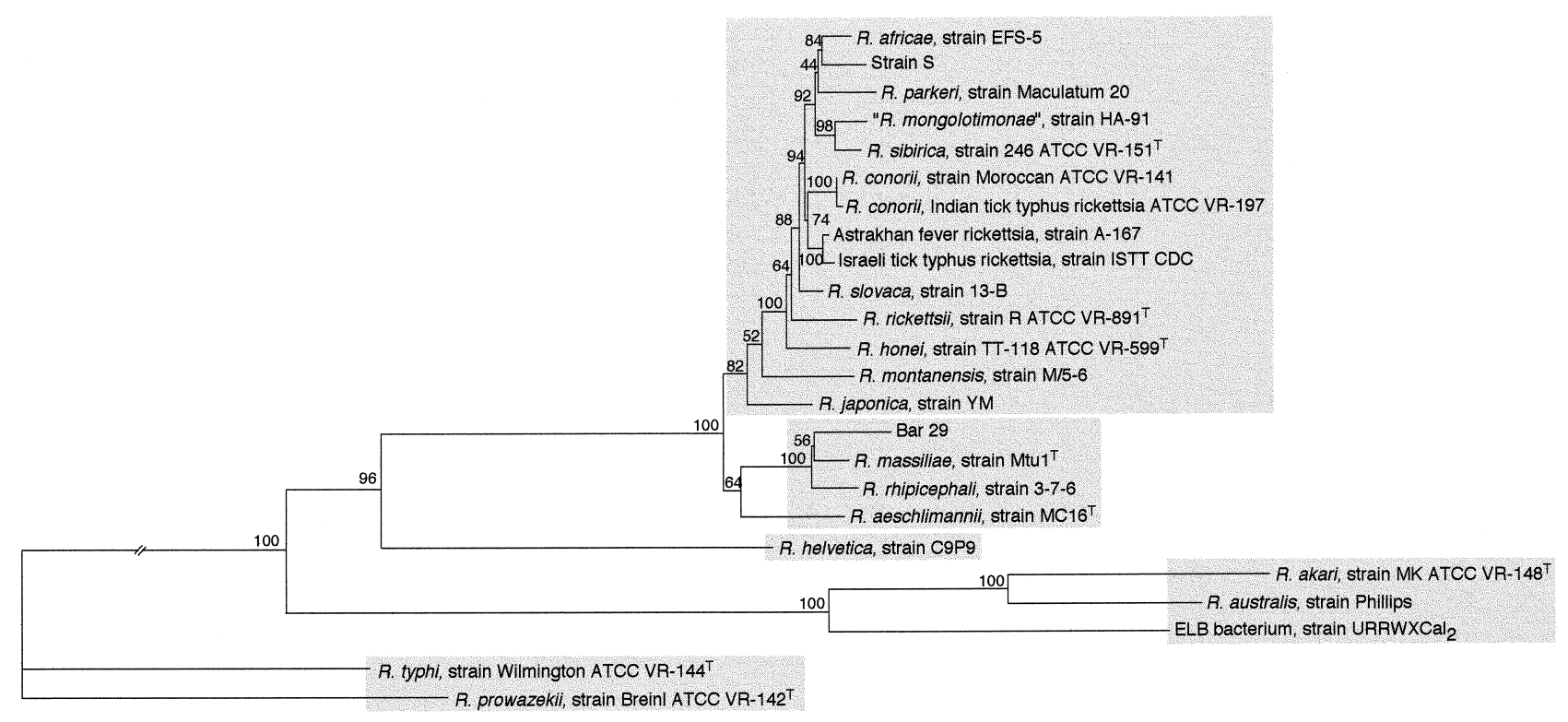

$1 \%$ difference

Fig. 1. Phylogenetic tree of the bacteria belonging to the genus Rickettsia inferred from comparison of the 'gene $D$ ' sequences. The evolutionary distance values were determined by the method of Kimura, and the tree was constructed by the neighbour-joining method. The numbers at nodes are proportions of 100 bootstrap resamplings that support the topology shown.

patterns. The two representatives of the TG, $R$. prowazekii and $R$. typhi, clustered together. R. akari, $R$. australis and the ELB bacterium clustered together and formed the R. akari group. Another distinct cluster contained $R$. massiliae, Bar 29, R. rhipicephali and $R$. aeschlimannii. $R$. helvetica was alone on a separate branch between the TG and the R. akari group in the parsimony and maximum-likelihood analyses and between the $R$. akari and $R$. massiliae groups in the distance matrices analysis. The $R$. rickettsii group was a large cluster containing 14 representatives: $R$. africae, strain $\mathrm{S}, R$. parkeri, $R$. sibirica, ' $R$. mongolotimonae', the $R$. conorii complex ( $R$. conorii strains, Astrakhan fever rickettsia and Israeli tick typhus rickettsia), $R$. slovaca, $R$. rickettsii, $R$. honei, $R$. montanensis and $R$. japonica. In this group, the phylogenetic organization was the same when the neighbour-joining and the maximum-likelihood analyses were used (Fig. 1). Differences were, however, noted in the parsimony analysis. $R$. montanensis diverged before $R$. japonica and $R$. sibirica clustered with $R$. rickettsii between the $R$. conorii complex and another group including $R$. africae, strain $\mathrm{S}, R$. parkeri and ' $R$. mongolotimonae'. For a particular method of analysis, the tree inferred from the protein sequences was practically the same as the one obtained from the nucleic acid sequences. However, in the parsimony analysis, $R$. aeschlimannii clustered with $R$. montanensis between the $R$. massiliae group and the $R$. rickettsii group and in the neighbour-joining analysis $R$. slovaca clustered with the $R$. conorii complex.
Significant bootstrap values $(>70 \%)$ were obtained for most of the nodes. In the neighbour-joining analysis when phylogeny was inferred from protein sequences, the node where $R$. slovaca branched was not well-supported. The same observation was also noted for the node where $R$. massiliae and Bar 29 clustered and the node where $R$. aeschlimannii branched when phylogeny was inferred from DNA sequences and for the nodes where $R$. parkeri and $R$. montanensis diverged in phylogenies inferred from both DNA and protein sequences. In the parsimony analysis when phylogenies were inferred from both DNA and protein sequence comparison, nonsignificant values were obtained for the nodes where $R$. africae, $R$. parkeri, strain $\mathrm{S}$, ' $R$. mongolotimonae', $R$. rickettsii and $R$. sibirica branched. For phylogeny inferred from comparison of protein sequences no significant value was obtained for the node where $R$. montanensis and $R$. aeschlimannii clustered. In the maximum-likelihood analysis when phylogeny was inferred from DNA sequence comparison, nonsignificant values were obtained for the nodes where $R$. parkeri and $R$. montanensis branched.

\section{DISCUSSION}

It has been suggested that the method of choice to establish bacterial phylogeny is the comparison of different gene sequences (Olsen \& Woese, 1993). So, the division of rickettsial species into two phyla, the SFG and TG, is not supported by genomic data. The 

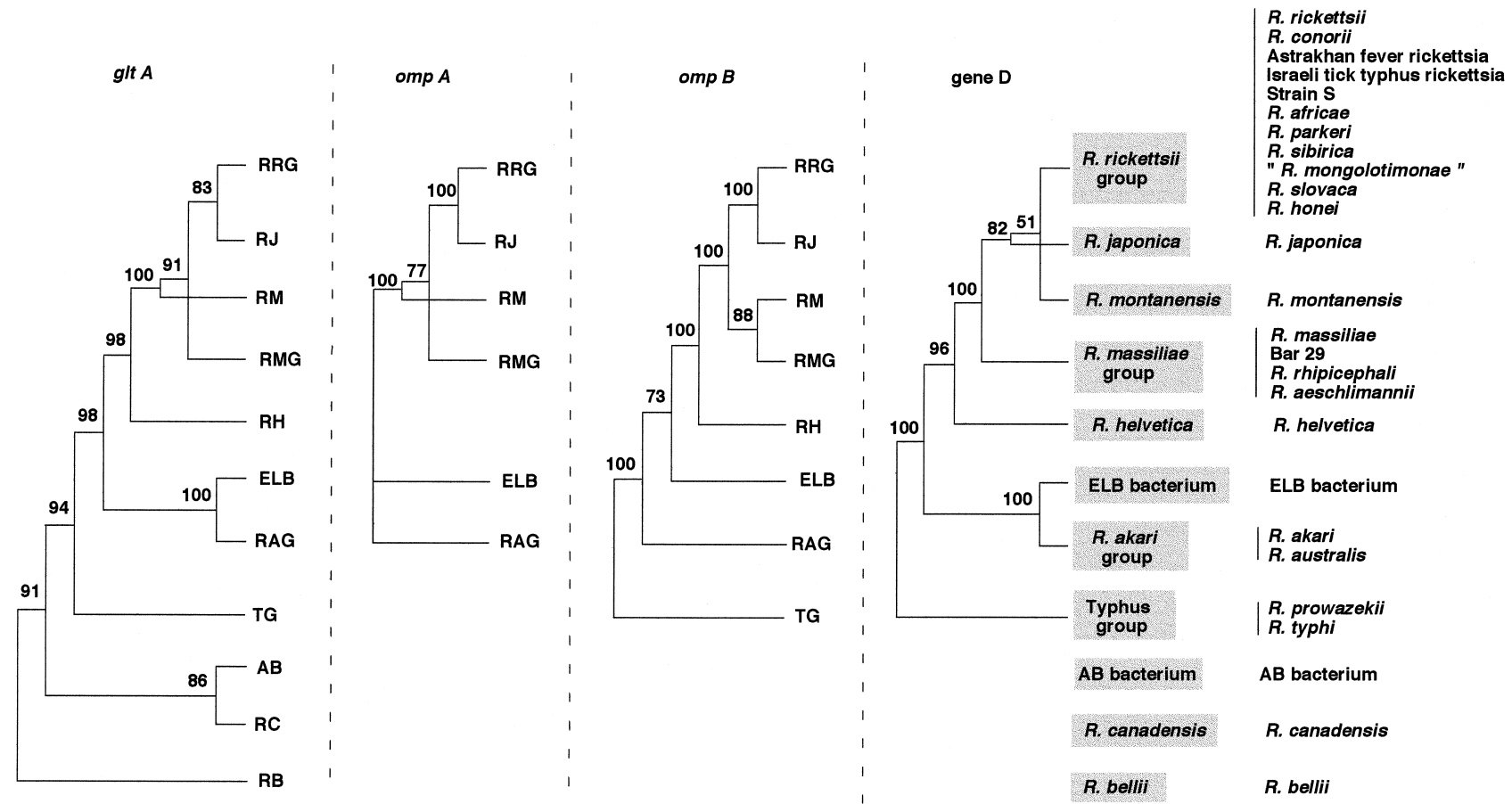

Fig. 2. Schematic representation of the dendrograms obtained by rickettsial sequence comparison of different genes. The phylogenetic organization is that obtained when the evolutionary distance values were determined by the method of Kimura and the tree was constructed by the neighbour-joining method. RRG, Rickettsia rickettsii group; RJ, $R$. japonica; $\mathrm{RM}, R$. montanensis; RMG, R. massiliae group; RH, R. helvetica; ELB, ELB bacterium; RAG, R. akari group; TG, typhus group; RC, $R$. canadensis; $\mathrm{RB}, R$. bellii.

16S rRNA gene is highly conserved in bacteria and phylogenetic analysis inferred from its sequence comparison can be a useful phylogenetic tool although it cannot be used to reliably classify Rickettsia spp. (Roux \& Raoult, 1995). For enteric bacteria, phylogeny studies based on rpoB gene sequences have been shown to be more reliable that those based on the $16 \mathrm{~S}$ rRNA gene (Mollet et al., 1997). In the SFG rickettsiae, bacteria included in the $R$. massiliae group, as defined on the basis of $о m p B$ gene sequence comparison ( $R$. montanensis, $R$. aeschlimannii, $R$. massiliae, Bar 29 and $R$. rhipicephali), were shown to be the only rifampin-resistant rickettsiae (Rolain et al., 1998). The outer-membrane protein genes omp $A$ and $o m p B$ were the more reliable tools to study the phylogeny of Rickettsia species.

In our experiments, we investigated the comparison of 'gene D' sequences as a tool to infer phylogeny of rickettsiae. The major discrepancy between our results and those previously reported for the glt $A, o m p A$ and omp $B$ genes was that $R$. montanensis clustered in the $R$. rickettsii group. Our study confirmed the presence of a TG with only two members, $R$. typhi and $R$. prowazekii, the vectors of which are fleas and body lice, respectively.

$R$. helvetica was found alone on a separate branch but its phylogenetic position was not stable and varied according to the phylogenetic analysis method used.
The $R$. akari group comprised three representatives: $R$. australis, transmitted by ticks; $R$. akari, transmitted by mites; and the flea-borne rickettsia ELB agent also named 'Rickettsia felis'. Previous studies based on sequence comparisons of genes encoding 16S rDNA and the $17 \mathrm{kDa}$ protein suggested that the ELB agent was within the TG (Radulovic et al., 1995; Higgins et al., 1996; Schiefer et al., 1994) but our results are in agreement with those obtained from the glt $A$ gene sequence comparison. The phylogenetic organization of the $R$. massiliae group was not as stable as those determined from omp $A$, omp $B$ and glt $A$ gene sequence comparison. In fact, significant bootstrap values were not always obtained and the inclusion of $R$. aeschlimannii in this group depended on the method of analysis used. However, $R$. montanensis was always excluded from this group.

In the $R$. rickettsii group, the $R$. conorii complex organization and the cluster $R$. africae/strain $\mathrm{S}$ were well established. The presence of the other representatives ( $R$. parkeri, ' $R$. mongolotimonae', $R$. sibirica, $R$. slovaca, $R$. rickettsii, $R$. honei, $R$. japonica and $R$. montanensis) in the $R$. rickettsii group was constant but comparison of their phylogenetic relationships was dependent on the method used.

We were not able to amplify 'gene $\mathrm{D}$ ' in $R$. canadensis and $R$. bellii, which have previously been shown to cluster with the AB bacterium (Werren et al., 1994) 
and with the PTB bacterium (Davis et al., 1998), respectively, when comparing 16S rDNA sequences (Roux \& Raoult, 1995; Stothard \& Fuerst, 1995). Comparison of the gene sequences encoding citrate synthase has also indicated that these two rickettsiae may be excluded from both the TG and SFG rickettsiae (Roux et al., 1997).

In attempting to establish a phylogeny of the Rickettsia, combination of the data obtained from different studies has indicated the need to reconsider the classification of these bacteria into more than two groups. The organization of the different clusters obtained from rickettsial sequence comparison of different genes is schematized in Fig. 2. Thirteen representatives of the genus Rickettsia are included in the $R$. rickettsii group: $R$. conorii strains, Astrakhan fever rickettsia, Israeli tick typhus rickettsia, $R$. sibirica, ' $R$. mongolotimonae', $R$. africae, strain $\mathrm{S}, R$. parkeri, $R$. slovaca, $R$. rickettsii, $R$. honei and $R$. japonica. A different arrangement inside this group was obtained when phylogeny was inferred from a comparison of different gene sequences. However, those obtained from omp $A$ and $о m p B$ analyses, which were identical, could correspond more closely to the true evolution of this group of the Rickettsia as significant bootstrap values were obtained for all the nodes in the three analysis methods (neighbourjoining, parsimony and maximum-likelihood) from both DNA and protein sequences. Moreover, where clustering differed between $g l t A$ and 'gene D' sequence comparisons, bootstrap values at the relevant nodes were not significant.

The phylogenetic position of $R$. montanensis is ambiguous. If we consider $g l t A$ and $o m p A$ analyses, it was outside the group including $R$. massiliae, Bar $29, R$. rhipicephali and $R$. aeschlimannii. The bootstrap values at the corresponding nodes were significant, being $100 \%$ in both reconstructions. However, if we consider отрB analysis, $R$. montanensis clustered inside this group, with a significant bootstrap value of $88 \%$. In 'gene D' analysis it clustered with the $R$. rickettsii group but the bootstrap value was not significant $(51 \%)$. More information is required to precisely determine the phylogenetic position of $R$. montanensis. However, the grouping of $R$. massiliae, Bar 29, $R$. rhipicephali and $R$. aeschlimannii is well established in the four analyses.

$R$. helvetica has followed a particular evolution and was always found alone on a separate branch, more often between the ELB bacterium and the group including $R$. massiliae. The phylogenetic position of the ELB bacterium is also ambiguous as either it clustered with the $R$. akari group ( $R$. akari and $R$. australis) (glt $A$ and 'gene $\mathrm{D}$ ' analyses) or it was alone on a separate branch (omp $B$ analysis). It seems that its preferential position is to cluster with the $R$. akari group as in the two cases bootstrap values are $100 \%$ compared with $73 \%$ in the $o m p B$ analysis, which is just above the limits of significance. $R$. akari and $R$. australis always clustered together, as did R. typhi and $R$. prowazekii, which formed the TG.

We suggest that $R$. canadensis, $R$. bellii and the AB bacterium were the first representatives of the genus Rickettsia to diverge from the common ancestor of this group of bacteria. Few sequences are available for these rickettsiae as often it was impossible to amplify DNA from the genes when amplification was carried out with consensus primers due to their major genomic divergence. More investigation will be particularly necessary to establish the phylogenetic relationships in this group.

Phylogenetic analyses of rickettsial species have advanced considerably during the last few years particularly due to the introduction of the molecular biology methodology. Nevertheless ambiguities remain. In the future, further information will be necessary to improve the phylogeny and establish a taxonomy of the genus Rickettsia.

\section{ACKNOWLEDGEMENTS}

We thank P. Kelly for the review of the manuscript.

\section{REFERENCES}

Anderson, B. E., Baumstark, B. R. \& Bellini, W. J. (1988). Expression of the gene encoding the 17-kilodalton antigen from Rickettsia rickettsii: transcription and posttranslation modification. J Bacteriol 170, 4493-4500.

Beati, L., Humair, P. F., Aeschlimann, A. \& Raoult, D. (1994). Identification of spotted fever group rickettsiae isolated from Dermacentor marginatus and Ixodes ricinus ticks collected in Switzerland. Am J Trop Med Hyg 51, 138-148.

Carl, M., Dobson, M. E., Ching, W.-M. \& Dasch, G. A. (1990). Characterization of the gene encoding the protective paracrystalline-surface-layer protein of Rickettsia prowazekii: presence of a truncated identical homolog in R. typhi. Proc Natl Acad Sci US A 87, 8237-8241.

Davis, M. J., Ying, Z., Brunner, B. R., Pantoja, A. \& Ferwerda, A. P. (1998). Rickettsial relative associated with Papaya bunchy top disease. Curr Microbiol 36, 80-84.

Dessen, P., Fondrat, C., Valencien, C. \& Munier, G. (1990). BISANCE: a French service to biomolecular databases. Cabios 6 , 355-356.

Eremeeva, M., Yu, X. \& Raoult, D. (1994). Differentiation among the spotted fever group rickettsiae species by analysis of restriction fragment length polymorphism of PCR-amplified DNA. J Clin Microbiol 32, 803-810.

Eremeeva, M. E., Balayeva, N. M., Roux, V., Ignatovich, V., Kotsinjan, M. \& Raoult, D. (1995). Genomic and proteinic characterization of strain $\mathrm{S}$, a rickettsia isolated from Rhipicephalus sanguineus ticks in Armenia. J Clin Microbiol 33, 2738-2744.

Felsenstein, J. (1989). PHYLIP - phylogeny inference package (version 3.2). Cladistics 5, 164-166.

Fournier, P. E., Roux, V. \& Roult, D. (1998). Phylogenetic analysis of the spotted fever rickettsiae by study of the outer surface protein rOmpA. Int J Syst Bacteriol 48, 839-849.

Higgins, J. A., Radulovic, S., Schriefer, M. E. \& Azad, A. F. (1996). 
Rickettsia felis: a new species of pathogenic rickettsia isolated from cat fleas. J Clin Microbiol 34, 671-674.

Kimura, M. (1980). A simple method for estimating evolutionary rate of base substitutions through comparative studies of nucleotide sequences. J Mol Evol 16, 111-120.

Mollet, C., Drancourt, M. \& Raoult, D. (1997). RpoB sequence analysis as a novel basis for bacterial identification. Mol Microbiol 26, 1005-1011.

Olsen, G. J. \& Woese, C. R. (1993). Ribosomal RNA: a key to phylogeny. FASEB J 7, 113-123.

Philip, R. N., Casper, E. A., Burgdorfer, W., Gerloff, R. K., Hughes, L. E. \& Bell, E. J. (1978). Serologic typing of rickettsiae of the spotted fever group by microimmunofluorescence. J Immunol 121, 1961-1968.

Radulovic, S., Higgins, J. A., Jaworski, D. C., Dasch, G. A. \& Azad, A. F. (1995). Isolation, cultivation, and partial characterization of the ELB agent associated with cat fleas. Infect Immun 63, 4826-4829.

Regnery, R. L., Spruill, C. L. \& Plikaytis, B. D. (1991). Genotypic identification of rickettsiae and estimation of intraspecies divergence for portions of two rickettsial genes. J Bacteriol 173, 1576-1589.

Rolain, J. M., Maurin, M., Vestris, G. \& Raoult, D. (1998). In vitro susceptibilities of 27 rickettsiae to 13 antimicrobials. Antimicrob Agents Chemother 42, 1537-1541.

Roux, V. \& Raoult, D. (1995). Phylogenetic analysis of the genus Rickettsia by $16 \mathrm{~S}$ rDNA sequencing. Res Microbiol 146, 385-396.

Roux, V. \& Raoult, D. (2000). Phylogenetic analysis of members of the genus Rickettsia using the gene encoding the outer membrane protein $\mathrm{rOmpB}($ omp B) . Int J Syst Evol Microbiol 50, 1449-1455.

Roux, V., Rydkina, E., Eremeeva, M. \& Raoult, D. (1997). Citrate synthase gene comparison, a new tool for phylogenetic analysis, and its application for the rickettsiae. Int J Syst Bacteriol 47, $252-261$
Saitou, N. \& Nei, M. (1987). The neighbor-joining method: a new method for reconstructing phylogenetic trees. Mol Biol Evol 4, 406-425.

Schiefer, M. E., Sacci, J. B. Jr, Dumler, J. S., Bullen, M. G. \& Azad, A. F. (1994). Identification of a novel rickettsial infection in a patient diagnosed with murine typhus. $J$ Clin Microbiol 32, 949-954.

Schuenke, K. W. \& Walker, D. H. (1994). Cloning, sequencing and expression of the gene coding for an antigenic 120-kilodalton protein of Rickettsia conorii. Infect Immun 62, 904-909.

Siebert, P. D., Chenchik, A., Kellogg, D. E., Lukyanov, K. A. \& Lukyanov, S. A. (1995). An improved PCR method for walking in uncloned genomic DNA. Nucleic Acids Res 23, 1087-1088.

Stenos, J., Roux, V., Walker, D. \& Raoult, D. (1998). Rickettsia honei sp. nov., the aetiological agent of Flinders Island spotted fever in Australia. Int J Syst Bacteriol 48, 1399-1404.

Stothard, D. R. \& Fuerst, P. A. (1995). Evolutionary analysis of the spotted fever and typhus groups of Rickettsia using $16 \mathrm{~S}$ rRNA gene sequences. Syst Appl Microbiol 18, 52-61.

Tamura, A., Ohashi, N., Urakami, H. \& Miyamura, S. (1995). Classification of Rickettsia tsutsugamushi in a new genus, Orientia gen. nov., as Orientia tsutsugamushi comb. nov. Int $J$ Syst Bacteriol 45, 589-591.

Uchiyama, T. (1997). Intracytoplasmic localization of antigenic heat-stable 120- to 130-kilodalton proteins (PS 120) common to spotted fever group rickettsiae demonstrated by immunoelectron microscopy. Microbiol Immunol 41, 815-818.

Uchiyama, T., Zhao, L. \& Uchida, T. (1996). Demonstration of a heat-stable 120-kilodalton protein of Rickettsia japonica as a spotted fever group-common antigen. Microbiol Immunol 40, 133-139.

Werren, J. H., Hurst, G. D., Zhang, W., Breeuwer, J. A. J., Stouthamer, R. \& Majerus, M. E. N. (1994). Rickettsial relative associated with male killing in the ladybird beetle (Adalia bipunctata). J Bacteriol 176, 388-394. 